\title{
Can the morning serum melatonin values reflect inflammatory activity: a biochemical evaluation?
}

\author{
Ibrahim Aydin
}

Received: 24 February 2014 / Accepted: 5 March 2014 / Published online: 17 March 2014

(C) Springer-Verlag Berlin Heidelberg 2014

We have read with great interest the article by Afkhamizadeh et al. [1] entitled "Morning melatonin serum values do not correlate with disease activity in rheumatoid arthritis: a cross-sectional study" in which they report morning serum melatonin levels are increased in rheumatoid arthritis, but these findings do not correlate with disease activity. In their paper, authors report that disease duration, season of sampling, medications, disease activity and gender did not influence morning melatonin levels. In this regard, we would like to make some additions to the paper in respect to interpretations of biochemical markers and study design.

Melatonin is a hormone found in animals, plants and microbes. In humans, melatonin is predominantly produced by the pineal gland and secreted into the blood. Melatonin secretion occurs in darkness especially makes a peak at 2-4 a.m. [2]. Production of melatonin is inhibited by light to the retina. It is principally blue light, around $460-480 \mathrm{~nm}$ wavelength that suppresses melatonin secretion in the morning [3]. After the secretion, $90 \%$ of melatonin is cleared from blood in a single passage through the liver. Because of these reasons, after dawn serum samples are unsuitable for evaluating the melatonin levels. The major hepatic metabolite of melatonin is 6-hydroxymelatonin sulphate (6-HMS) which excreted mainly in urine. It has been recognized that the amount of 6-HMS in urine is a good index of pineal gland activity for the desired period [4].

Melatonin is found in plants as mentioned above. When humans consume foods rich in melatonin such as banana, orange and pineapple, serum melatonin levels significantly increase [5]. In this study, participants' diet is not mentioned in the paper.

I. Aydin $(\bowtie)$

Sarikamis Military Hospital, Kars, Turkey

e-mail: mdiaydin@hotmail.com
Use of drugs is another confounding factor for this study. Previous studies showed that nonsteroidal antiinflammatory drugs such as ibuprofen and indomethacin suppress melatonin secretion and alter the circadian rhythm [6]. Patients with rheumatoid arthritis usually use these medicines. For evaluating the melatonin levels more accurately, information should be provided about drug use.

In conclusion, the explanation of these concerns will certainly provide the clearer information for the readers.

\section{References}

1. Afkhamizadeh M, Sahebari M, Seyyed-Hoseini SR (2014). Morning melatonin serum values do not correlate with disease activity in rheumatoid arthritis: a cross-sectional study. Rheumatol Int (Epub ahead of print). doi:10.1007/s00296-013-2930-x

2. Pandi-Perumal SR, Sirinivasan V, Maestroni GJ, Cardinali DP, Poeggeler B, Hardeland R (2006) Melatonin: nature's most versatile biological signal? FEBS J 273:2813-2838

3. Brainard GC, Hanifin JP, Greeson JM, Byrne B, Glickman G, Gerner E, Rollag MD (2001) Action spectrum for melatonin regulation in humans: evidence for a novel circadian photoreceptor. J Neurosci 21(16):6405-6412

4. Ozkan E, Yaman H, Cakir E, Deniz O, Oztosun M, Gumus S, Akgul EO, Agilli M, Cayci T, Kurt YG, Aydin I, Arslan Y, Ilhan N, Ilhan N, Erbil MK (2012) Plasma melatonin and urinary 6-hydroxymelatonin levels in patients with pulmonary tuberculosis. Inflammation 35(4):1429-1434

5. Sae-Teaw M, Johns J, Johns NP, Subongkot S (2012) Serum melatonin levels and antioxidant capacities after consumption of pineapple, orange, or banana by healthy male volunteers. J Pineal Res 55(1):58-64

6. Murphy PJ, Myers BL, Badia P (1996) Nonsteroidal anti-inflammatory drugs alter body temperature and suppress melatonin in humans. Physiol Behav 59(1):133-139 